\title{
Assessing the ability of DFT methods to describe static electron correlation effects: CO core level binding energies as a representative case
}

Noèlia Pueyo Bellafont, Paul S. Bagus, Carmen Sousa, and Francesc Illas

Citation: The Journal of Chemical Physics 147, 024106 (2017); doi: 10.1063/1.4991833

View online: https://doi.org/10.1063/1.4991833

View Table of Contents: http://aip.scitation.org/toc/jcp/147/2

Published by the American Institute of Physics

\section{Articles you may be interested in}

Coupled cluster valence bond theory for open-shell systems with application to very long range strong correlation in a polycarbene dimer

The Journal of Chemical Physics 147, 024107 (2017); 10.1063/1.4991797

Communication: DFT treatment of strong correlation in 3d transition-metal diatomics

The Journal of Chemical Physics 146, 211105 (2017); 10.1063/1.4985084

Strong correlation in incremental full configuration interaction

The Journal of Chemical Physics 146, 224104 (2017); 10.1063/1.4985566

Low-scaling first-order properties within second-order Møller-Plesset perturbation theory using Cholesky decomposed density matrices

The Journal of Chemical Physics 147, 024101 (2017); 10.1063/1.4990413

Time-dependent $\mathrm{N}$-electron valence perturbation theory with matrix product state reference wavefunctions for large active spaces and basis sets: Applications to the chromium dimer and all-trans polyenes

The Journal of Chemical Physics 146, 244102 (2017); 10.1063/1.4986975

A note on the accuracy of KS-DFT densities

The Journal of Chemical Physics 147, 204103 (2017); 10.1063/1.5001939

\section{AIP | The Jounal of Chemical Physics}




\title{
Assessing the ability of DFT methods to describe static electron correlation effects: $\mathrm{CO}$ core level binding energies as a representative case
}

\author{
Noèlia Pueyo Bellafont, ${ }^{1}$ Paul S. Bagus,${ }^{2}$ Carmen Sousa, ${ }^{1}$ and Francesc Illas ${ }^{1}$ \\ ${ }^{1}$ Departament de Ciència de Materials i Química Física \& Institut de Química Teòrica i Computacional \\ (IQTCUB), Universitat de Barcelona, C/Martí i Franquès 1, 08028 Barcelona, Spain \\ ${ }^{2}$ Department of Chemistry, University of North Texas, Denton, Texas 76203-5017, USA
}

(Received 24 April 2017; accepted 23 June 2017; published online 13 July 2017)

\begin{abstract}
We use a total energy difference approach to explore the ability of various density functional theory based methods in accounting for the differential effect of static electron correlation on the $\mathrm{C}(1 \mathrm{~s})$ and $\mathrm{O}(1 \mathrm{~s})$ core level binding energies (BEs) of the $\mathrm{CO}$ molecule. In particular, we focus on the magnitude of the errors of the computed $\mathrm{C}(1 \mathrm{~s})$ and $\mathrm{O}(1 \mathrm{~s}) \mathrm{BEs}$ and on their relative difference as compared to experiment and to previous results from explicitly correlated wave functions. Results show that the different exchange-correlation functionals studied here behave rather erratically and a considerable number of them lead to large errors in the BEs and/or the BE shifts. Nevertheless, the TPSS functional, its TPSSm and RevTPSS derivations, and its corresponding hybrid counterpart, TPSSh, perform better than average and provide BEs and BE shifts in good agreement with experiment. Published by AIP Publishing. [http://dx.doi.org/10.1063/1.4991833]
\end{abstract}

\section{INTRODUCTION}

X-Ray Photoelectron Spectroscopy, XPS, is a widely used method to study the composition, properties, and electronic structure of a very wide range of materials from gas phase atoms and molecules to condensed phase systems. For early references for the application of XPS, in particular using laboratory sources for the X-rays used to ionize the sample studied, see Refs. 1 and 2. There have been modern developments, including the use of higher pressures, approaching ambient pressure, and the use of hard X-rays, which further extend the power of XPS to determine materials properties; see, for example, Ref. 3. For such studies, it is important to analyze the features of the photoionized electrons, in particular, the corelevel binding energies, BEs. The features of interest include the shifts of BEs, $\triangle B E s$, between the same atom in different environments and between different atoms and the number and energy spacing of main and satellite features for core-level BEs. The theory has played a major role in the interpretation of these features of XPS BEs especially to relate them to the electronic properties of the systems studied; for an early review, see Ref. 4; see also Refs. 5 and 6 and a more recent review. ${ }^{7}$ It has been shown that the electronic structure obtained both from Hartree-Fock (HF) and from Density functional theory (DFT) can predict the core-level BEs of molecules containing light atoms with a high degree of accuracy; see Refs. 8-10 and references therein. These references also present an analysis of the accuracy of the BEs obtained using different DFT functionals.

It is also known that there is an important set of electron correlations, or many-body effects, involving near degeneracies among different orbitals that contribute significantly to the XPS BE features; see Ref. 7 and references therein and Ref. 11. These many-body effects are often described as static correlation effects to distinguish them from the dynamic correlation effects that are needed to obtain very accurate energies and energy differences. ${ }^{12-14}$ Because there are questions regarding the appropriate methods for treating static correlation effects within DFT, ${ }^{15,16}$ it is necessary to establish the accuracy and reliability of the DFT treatment and calculation of core-level BEs when static correlation is important. A previous study showed that inclusion of static electron correlation effects led to significant changes in the $\mathrm{C}(1 \mathrm{~s})$ and $\mathrm{O}(1 \mathrm{~s})$ core level binding energies of the $\mathrm{CO}$ molecule. ${ }^{11}$ Motivated by the importance of this well-defined physical effect, a systematic study has been carried out to investigate whether different DFT based methods are able to properly treat the physical effects responsible for the static correlation contributions to core level BEs.

For the study of the DFT treatment of static correlation, we have chosen the ideal case of the $\mathrm{C}(1 \mathrm{~s})$ and $\mathrm{O}(1 \mathrm{~s})$ BEs of the $\mathrm{CO}$ molecule. ${ }^{11}$ This is an ideal case in the sense that the static correlation is different for the initial, ground state of neutral $\mathrm{CO}$ and for the $\mathrm{C}(1 \mathrm{~s})$ and $\mathrm{O}(1 \mathrm{~s})$ hole configurations for the final states after core-level ionization. The static correlation for the initial and final state configurations arises from mixing of occupations of the bonding $1 \pi$ and antibonding $2 \pi$ orbitals ${ }^{11}$ where for HF wave functions, $1 \pi$ is fully occupied and $2 \pi$ is empty. That the contribution of $2 \pi$ might be different can be understood in terms of the equivalent core model of Jolly ${ }^{17,18}$ where the core ionized atom is replaced with one element higher in the periodic table. Thus $\mathrm{CO}$ with a $\mathrm{C}(1 \mathrm{~s})$ hole is equivalent to $\mathrm{NO}^{+}$and $\mathrm{CO}$ with a $\mathrm{O}(1 \mathrm{~s})$ hole is equivalent to $\mathrm{CF}^{+}$and one should not expect the static correlation to be the same for $\mathrm{CO}, \mathrm{NO}^{+}$, and $\mathrm{CF}^{+}$ and indeed it is not. ${ }^{11}$ Since we are concerned with manybody effects, we define BEs as the difference between the variationally determined total energies for the initial state configuration and the final, core-hole state configuration. These BEs are commonly denoted as $\mathrm{BE}(\triangle \mathrm{SCF})$ and are given 
by

$$
\mathrm{BE}(\Delta \mathrm{SCF})=\mathrm{E}(\text { ion })-\mathrm{E}(\text { Ground State }),
$$

where $\mathrm{E}$ (ion) and $\mathrm{E}$ (Ground State) are the variational $\mathrm{HF}$ or DFT total energies. It is to be recalled that Koopmans' theorem (KT) BEs obtained using HF or DFT orbital energies give rather poor values for core level $\mathrm{BEs}$; for the $\mathrm{KT} \mathrm{O}(1 \mathrm{~s}) \mathrm{BE}$ of CO, see Table 2 of Ref. 7 . Thus we use only $\operatorname{BE}(\Delta S C F)$. For similar reasons, we avoid the use of the generalized transition state BEs proposed by Chong. ${ }^{19-21}$

In our previous study of the consequences of static correlation for core level BEs, ${ }^{11}$ we have shown that they can lead to unexpected errors in the theoretical predictions of core-level BEs. To place our work on the DFT predictions for BEs where static correlation is critically important, we briefly review the analysis of the effect of static correlation on these BEs. From arguments about the importance of correlation being proportional to the number of electron pairs, expected to be true for dynamic correlation, then the $\mathrm{HFBE}(\triangle \mathrm{SCF})$ should be smaller than the experimental $\mathrm{BE}$ since the correlation is smaller in the ion. ${ }^{22}$ Thus, one would expect that, when relativistic corrections are included, the $\mathrm{HF} \mathrm{BE}(\triangle \mathrm{SCF})$ would be smaller than experiment by $\sim 0.25-0.50 \mathrm{eV}^{8-10}$ However, it is found that for $\mathrm{CO}$, the $\mathrm{C}(1 \mathrm{~s}) \mathrm{BE}(\triangle \mathrm{SCF})$, with relativistic corrections, is larger than experiment by over $1 \mathrm{eV} .{ }^{11}$ In other words, the correlation error for the $\mathrm{CO}$ ion where a $\mathrm{C}(1 \mathrm{~s})$ electron has been removed is larger, by more than an $\mathrm{eV}$, than for neutral $\mathrm{CO}$, an unexpected result. Another way of viewing the accuracy of the calculation of the BEs is to consider the BE shift between $\mathrm{BE}[\mathrm{O}(1 \mathrm{~s})]$ and $\mathrm{BE}[\mathrm{C}(1 \mathrm{~s})]$ where the shift, $\triangle \mathrm{BE}$, is defined as

$$
\Delta \mathrm{BE}=\mathrm{BE}[\mathrm{O}(1 \mathrm{~s})]-\mathrm{BE}[\mathrm{C}(1 \mathrm{~s})] .
$$

If $\triangle \mathrm{BE}$ calculated with $\mathrm{BE}(\triangle \mathrm{SCF})$, using $\mathrm{HF}$, and the experimental $\triangle \mathrm{BE}$ are the same, then the theoretical treatment of the two ions have the same errors and give a balanced treatment. This is relevant for the analysis of experiment since one often knows that the BE shifts more accurately than the absolute values of the BEs with reference to the vacuum zero of energy, especially for condensed systems. ${ }^{5}$ Indeed, to our knowledge for the first time, we shall use the $\triangle \mathrm{BE}$ criterion as a measure of the quality of the theoretical treatments of the holestate ions of CO. From the data in Ref. 11, the error of $\triangle \mathrm{BE}$ from the $\mathrm{HF}$ wave functions is over $1.5 \mathrm{eV}$ indicating a serious imbalance of the accuracy of theoretical HF treatment of the different hole states. When the $1 \pi-2 \pi$ static many-body effects are treated with Complete Active Space SCF (CASSCF) methods $^{23}$ where the $4 \mathrm{CO} \pi$ electrons are distributed in all possible ways over the four $1 \pi_{x}, 1 \pi_{y}, 2 \pi_{x}$, and $2 \pi_{y}$ orbitals, denoted as CAS $(4,4)$, the results are quite different. In particular, $\triangle \mathrm{BE}$ obtained from the $\operatorname{CAS}(4,4) \mathrm{BE}(\triangle \mathrm{SCF})$ values ${ }^{11}$ is within $0.01 \mathrm{eV}$ of experiment clearly indicating that the CASSCF treatment of static electron correlation is balanced and accurate.

From the previous discussion, it is clear that the core level $\mathrm{BEs}$ of $\mathrm{CO}$ provide an excellent test on the ability of the different DFT methods to include static electron correlation effects. However, distinction between dynamic and non-dynamic electron correlation effects in DFT is not straightforward. ${ }^{24}$ It is usually argued that DFT includes mainly short range exchange and Coulomb correlation effects, i.e., mainly of the dynamic correlation type. However, it is not clear that static correlation effects, which are related to near-degeneracy between different electronic states and, therefore, system specific, are properly taken into account. ${ }^{15,16}$ In the present work, we analyze the performance of various DFT methods, differing in the form of the exchange-correlation $(x c)$ functional, on determining the correlation effects (static and dynamic) that are essential to obtain accurate computed $\mathrm{C}(1 \mathrm{~s})$ and $\mathrm{O}(1 \mathrm{~s})$ core hole BEs of the $\mathrm{CO}$ molecule, as well as their relative difference. Various $\mathrm{xc}$ functionals belonging to the different families of approximations, essentially, local density approach (LDA), generalized gradient approach (GGA), and meta-GGA, and hybrid functionals containing a part of non-local Fock exchange and range-separated hybrid methods, will be studied. Each of these levels of approximation (LDA, GGA, meta-GGA, hybrid, etc.) is often identified as a rung in terms of Jacob's ladder scheme as defined by Perdew and Schmidt ${ }^{25}$ where the exact result will be at the topmost rung. During the last thirty years, a large number of functionals have been proposed and a lot of effort is still invested in the development of new functionals. ${ }^{26,27}$ This development is usually carried out either by comparing to experimental datasets involving as many properties as possible $^{26}$ or by relying on more fundamental properties of the unknown exact xc functional. ${ }^{27}$ The present work is not aimed at just investigating the accuracy of different functionals in predicting core level binding energies of the $\mathrm{CO}$ molecule, rather it introduces a new well-defined physical effect that may be used to verify whether the right value is obtained from the right reason. In particular, the present results allow one to check if general conclusions can be raised for each level of approximation or instead, each particular functional behaves independently.

\section{COMPUTATIONAL DETAILS}

The $\mathrm{C}(1 \mathrm{~s})$ and $\mathrm{O}(1 \mathrm{~s})$ core level BEs of the CO molecule have been obtained from $\triangle \mathrm{SCF}$ calculations by taking the total energy difference between the ionized core-hole ${ }^{2} \Sigma^{+}$state and the ground closed shell state of ${ }^{1} \Sigma^{+}$symmetry, as explained in detail in previous works ${ }^{8-10}$ and using the set of different exchange-correlation functionals described below.

Calculations have been carried out using a large totally uncontracted Gaussian basis set for both $\mathrm{C}$ and $\mathrm{O}$ atoms. This ensures the required flexibility to accurately describe both the initial neutral state and the final ionized system. An uncontracted Partridge (14s 9p) basis set ${ }^{28}$ has been used for $\mathrm{C}$ and $\mathrm{O}$ atoms, augmented by a single polarization $\mathrm{d}$ function with exponent 0.80 for $\mathrm{C}$ and 1.28 for $\mathrm{O}$.

DFT calculations, within the standard Kohn-Sham implementation, using different exchange-correlation functionals have been performed. Results obtained by means of HF and CAS $(4,4)$ calculations have been previously reported ${ }^{11}$ using a different but still very large basis set. Nevertheless, to allow a more direct comparison, results from HF and CAS $(4,4)$ obtained using the same basis set as in the DFT calculations are also reported for comparison; in addition, these HF and CAS use $\mathrm{C}-\mathrm{O}$ distances consistent with the choices for the DFT calculations. All calculations are non-relativistic and 
have been carried out in a spin restricted approach. However, for all cases, we have added a rigorous relativistic correction. ${ }^{11}$ From the large list of DFT methods available, we have selected some of them as representatives of the different families. Three different functionals related to the GGA implementation of the exchange-correlation potential have been chosen; these are the well-known implementation by Perdew, Burke, and Ernzerhof (PBE), ${ }^{29}$ and its modification aimed at providing a better description of lattice properties of solid structures (PBEsol) ${ }^{30}$ and the second-order generalized gradient approximation (SOGGA11) functional proposed by Peverati, Zhao, and Truhlar. ${ }^{31}$ Four functionals belonging to the meta-GGA family have also been studied. Those include two functionals of the Minnesota family, the M06$\mathrm{L}^{32}$ and M11- $\mathrm{L}^{33}$ functionals, and three versions of the Tao, Perdew, Staroverov, Scuseria functionals, TPSS, ${ }^{34}$ TPSSm, ${ }^{35}$ and RevTPSS. ${ }^{36}$ Six hybrid functionals, incorporating various degrees of Hartree-Fock exchange, have been considered. Three of them are classified as hybrid GGA functionals, the Becke 3-parameter exchange ${ }^{37}$ Lee, Yang, and Parr correlation functional, ${ }^{38}$ B3LYP, with $20 \%$ of HF exchange, PBE0 ${ }^{39}$ (25\% of exact exchange), and SOGGA11- $\mathrm{X}^{40}$ (35.42\% of exact exchange). Three hybrid meta-GGA functionals have also been taken into consideration, the TPSSh${ }^{41}$ functional (10\% of exact exchange included) and Minnesota M06 and M06-2X with 27\% and 54\% of Hartree-Fock exchange, respectively. ${ }^{42}$ Finally, we consider the M11 range-separated hybrid functional, ${ }^{43}$ with exact exchange varying from $42.8 \%$ in the short-range to $100 \%$ in the long-range interelectronic separation.

The equilibrium geometry of the $\mathrm{CO}$ molecule is optimized for the neutral ground state for each particular method. The BEs are computed as vertical transitions which is justified given the time scale of core level ionization in the XPS experiments. A 4th degree polynomial fit to the potential energy curve is used to extract the harmonic vibrational frequency, $\omega_{\mathrm{e}}$, the anharmonic correction, $\omega_{\mathrm{e}} \chi_{\mathrm{e}}$, vibrational-rotational coupling, and centrifugal distortion terms ${ }^{44}$ which are also compared to experiment. It is noted that the values of the HF and CAS BEs taken from Ref. 11 are for calculations at the correct experimental $\mathrm{C}-\mathrm{O}$ distance; this is different from the calculations presented in this paper which are all for calculated equilibrium $\mathrm{C}-\mathrm{O}$ distances for each method considered. Thus the values presented in this paper for the CAS $(4,4)$ BEs will be somewhat different from those discussed above and given in Ref. 9; these differences are $\sim 0.1 \mathrm{eV}$. The origin of these differences in terms of the character of the $\mathrm{CO}$ chemical bond will be discussed further in a forthcoming paper. ${ }^{45}$ DFT and HF calculations have been performed with the GAMESS-06 program,${ }^{46}$ and the CASSCF calculations have been carried out using MOLCAS. ${ }^{47}$

\section{RESULTS AND DISCUSSION}

We start the discussion by analyzing the results obtained for the $\mathrm{C}-\mathrm{O}$ interatomic distance and the harmonic vibrational frequency reported in Table I, which also collects experimental results. ${ }^{48}$ The $\mathrm{C}-\mathrm{O}$ distance has been optimized for each one of the methods explored in this work. As it is well known,
TABLE I. Experimental and computed $\mathrm{C}-\mathrm{O}$ interatomic equilibrium distance (in $\AA$ ) and harmonic vibrational frequency $\left(\omega_{\mathrm{e}}\right.$ in $\mathrm{cm}^{-1}$ ) through HartreeFock (HF), CASSCF $[\mathrm{CAS}(4,4)]$, and various density functional theory (DFT) based methods. Errors calculated as the difference between the computed and experimental values are reported for the $\mathrm{C}-\mathrm{O}$ distance and the harmonic vibrational frequency.

\begin{tabular}{lcccc}
\hline \hline & $\mathrm{R}_{\mathrm{e}}(\AA)$ & Error $(\AA)$ & $\omega_{\mathrm{e}}\left(\mathrm{cm}^{-1}\right)$ & Error $\left(\mathrm{cm}^{-1}\right)$ \\
\hline Experiment $^{48}$ & 1.128 & $\ldots$ & 2170 & $\ldots$ \\
\hline HF & 1.103 & -0.025 & 2437 & 267 \\
CAS(4,4) & 1.118 & -0.010 & 2314 & 144 \\
\hline PBE & 1.137 & 0.009 & 2131 & -39 \\
PBEsol & 1.135 & 0.007 & 2147 & -23 \\
SOGGA11 & 1.129 & 0.001 & 2167 & -3 \\
\hline M06-L & 1.129 & 0.001 & 2204 & 34 \\
M11-L & 1.119 & -0.009 & 2234 & 64 \\
TPSS & 1.134 & 0.006 & 2138 & -32 \\
TPSSm & 1.137 & 0.009 & 2133 & -37 \\
RevTPSS & 1.132 & 0.004 & 2154 & -16 \\
\hline B3LYP & 1.126 & -0.002 & 2213 & 43 \\
PBE0 & 1.124 & -0.004 & 2244 & 74 \\
SOGGA11-X & 1.124 & -0.004 & 2273 & 103 \\
\hline TPSSh & 1.131 & 0.003 & 2184 & 14 \\
M06 & 1.124 & -0.004 & 2243 & 2286 \\
M06-2X & 1.120 & -0.008 & 2271 & 73 \\
\hline M11 & 1.121 & -0.007 & & 14 \\
\hline \hline & & & & \\
\hline
\end{tabular}

geometries obtained from DFT methods are, in general, in better agreement with experiment than HF geometries, since in HF electron correlation effects are not taken into account. Inclusion of static electron correlation due to near degeneracy by means of CAS $(4,4)$ calculations improves the accuracy of the $\mathrm{C}-\mathrm{O}$ distance compared to HF. The difference with experiment, of $-0.010 \AA$, is due to the lack of dynamical electron correlation effects. Overall, all DFT methods studied in this work provide accurate equilibrium distances, in better agreement with experiment than HF or CAS $(4,4)$ values. As shown in Table I, in general, hybrid methods give distances somewhat shorter than experiment while distances obtained by methods based on the GGA approximation are larger than experiment by a similar amount. In any case, the errors with respect to the experimental value are rather small, less than one hundredth of an angstrom for all DFT methods studied. For comparison, vibro-rotational spectroscopic constants are provided in Table $\mathrm{S} 1$ of the supplementary material. DFT computed $\omega_{\mathrm{e}}$ are within a maximum error of approximately $\pm 100 \mathrm{~cm}^{-1}$ compared to experiment, also in better accord with experimental data than the values obtained through $\mathrm{HF}$ or $\operatorname{CAS}(4,4)$ wave function calculations.

Results for the $\mathrm{O}(1 \mathrm{~s})$ and $\mathrm{C}(1 \mathrm{~s})$ core level BEs computed as explained in Sec. II are collected in Table II. The error reported is defined as the difference between the calculated and experimental ${ }^{49} \mathrm{BEs}$ and, consequently, a negative value indicates that the calculated $\mathrm{BE}$ is smaller than experiment. Results for the error of the $\mathrm{O}(1 \mathrm{~s})$ core level BEs with respect to experiment are reported in Table II whereas the absolute values of the computed BEs are reported in Table $\mathrm{S} 2$ of the 
TABLE II. Errors in the $\mathrm{O}(1 \mathrm{~s})$ and $\mathrm{C}(1 \mathrm{~s})$ core level binding energies and $\mathrm{BE}$ shifts $(\triangle \mathrm{BE})$ for the $\mathrm{CO}$ molecule as obtained from $\triangle \mathrm{SCF}$ calculations using Hartree-Fock (HF), CASSCF [CAS $(4,4)]$ and various density functional theory (DFT) based methods. The error is the difference between the computed BEs or $\triangle \mathrm{BEs}$ and the experimental measures. Error-rel denotes the error including a correction for relativistic effects (see the text). All values are in $\mathrm{eV}$.

\begin{tabular}{|c|c|c|c|c|c|c|}
\hline \multirow{3}{*}{ Experiment ${ }^{49}$} & \multicolumn{2}{|c|}{$\mathrm{O}(1 \mathrm{~s}) \mathrm{BE}$} & \multicolumn{2}{|c|}{$\mathrm{C}(1 \mathrm{~s}) \mathrm{BE}$} & \multicolumn{2}{|c|}{$\triangle \mathrm{BE}$} \\
\hline & \multicolumn{2}{|c|}{$542.57 \pm 0.03$} & \multicolumn{2}{|c|}{$296.24 \pm 0.03$} & \multicolumn{2}{|c|}{$246.33 \pm 0.06$} \\
\hline & Error & Error-rel & Error & Error-rel & Error & Error-rel \\
\hline $\mathrm{HF}$ & -0.91 & -0.46 & 0.66 & 0.79 & -1.57 & -1.25 \\
\hline $\operatorname{CAS}(4,4)$ & -0.76 & -0.31 & -0.55 & -0.42 & -0.21 & 0.11 \\
\hline PBE & -1.19 & -0.74 & -0.71 & -0.58 & -0.48 & -0.16 \\
\hline PBEsol & -3.20 & -2.75 & -2.21 & -2.08 & -0.99 & -0.67 \\
\hline SOGGA11 & 0.03 & 0.48 & 0.36 & 0.49 & -0.33 & -0.01 \\
\hline M06-L & -0.28 & 0.17 & -0.33 & -0.20 & 0.05 & 0.37 \\
\hline M11-L & 0.56 & 1.01 & 0.49 & 0.62 & 0.07 & 0.39 \\
\hline TPSS & -0.36 & 0.09 & 0.08 & 0.21 & -0.44 & -0.12 \\
\hline TPSSm & -0.43 & 0.02 & 0.04 & 0.17 & -0.47 & -0.15 \\
\hline RevTPSS & -0.04 & 0.41 & 0.29 & 0.42 & -0.33 & -0.01 \\
\hline B3LYP & -0.47 & -0.02 & 0.35 & 0.48 & -0.82 & -0.50 \\
\hline PBE0 & -0.91 & -0.46 & -0.19 & -0.06 & -0.72 & -0.40 \\
\hline SOGGA11-X & -0.63 & -0.18 & 0.28 & 0.41 & -0.91 & -0.59 \\
\hline TPSSh & -0.33 & 0.12 & 0.21 & 0.34 & -0.54 & -0.22 \\
\hline M06 & -0.96 & -0.51 & -0.30 & -0.17 & -0.66 & -0.34 \\
\hline M06-2X & -0.94 & -0.49 & 0.32 & 0.45 & -1.26 & -0.94 \\
\hline M11 & 0.20 & 0.65 & 0.73 & 0.86 & -0.53 & -0.21 \\
\hline
\end{tabular}

supplementary material. As commented before, the $\mathrm{O}(1 \mathrm{~s})$ $\triangle \mathrm{SCF} \mathrm{BE}$ computed employing the $\mathrm{HF}$ method is, as expected, smaller than experiment, by $0.91 \mathrm{eV}$. The relativistic corrections on the BEs have been determined by means of fourcomponent Dirac-Hartree-Fock calculations ${ }^{11}$ on $\mathrm{O}$ and $\mathrm{C}$ atoms. We have shown that the use of corrections from atomic BEs is extremely reliable for the CO molecule by comparing these atomic estimates with direct relativistic calculations for the CO BEs; see Ref. 11. The relativistic corrections from the atomic calculations are nearly identical with those from the exact relativistic calculation on the $\mathrm{CO}$ molecule. ${ }^{11}$ For the $\mathrm{C}(1 \mathrm{~s})$ and $\mathrm{O}(1 \mathrm{~s})$ core holes, relativistic effects increase the $\mathrm{BE}$ by 0.13 and $0.45 \mathrm{eV}$, respectively. Hence, when relativistic corrections are included, the error in the $\mathrm{O}(1 \mathrm{~s}) \mathrm{BE}$ is reduced to $0.46 \mathrm{eV}$. The sign or the error and the magnitude suggest that static electron correlation will not have a very large impact on the $\mathrm{O}(1 \mathrm{~s}) \mathrm{BE}$ as will be confirmed below. Indeed, when static correlation contributions are allowed for by means of CAS $(4,4)$ calculations, only a modest change of the BE is observed compared to HF; BE becomes $0.15 \mathrm{eV}$ closer to the experiment. This is about half of the remaining error of $0.31 \mathrm{eV}$ for the relativistic corrected CAS $(4,4) \mathrm{O}(1 \mathrm{~s}) \mathrm{BE}$. The CAS $(4,4)$ value is underestimated compared to experiment due to missing dynamical electron correlation.

For the C(1s) BEs, Table II shows a very different behavior than for the $\mathrm{O}(1 \mathrm{~s})$ BEs. Here, as mentioned above and contrary to what should be expected, HF $\triangle \mathrm{SCF} B E$ is larger than experiment by $0.66 \mathrm{eV}$ and by $0.79 \mathrm{eV}$ when relativistic effects are taken into account, showing clearly that the electron correlation in the final ionized state is larger than in the initial neutral state which is unexpected and suggests that static electron correlation effects could play an important role for the $\mathrm{C}(1 \mathrm{~s}) \mathrm{BE} .{ }^{11}$ In fact, in agreement with previous work, ${ }^{11}$ inclusion of near degeneracy of the $\mathrm{CO}(1 \pi)$ and $\mathrm{CO}(2 \pi)$ valence levels in the calculation of the $\mathrm{C}(1 \mathrm{~s}) \mathrm{BE}$ by means of CAS $(4,4)$ calculations reverses the sign of the error which now becomes $-0.55 \mathrm{eV}$ and $-0.42 \mathrm{eV}$ with relativistic corrections, i.e., the computed $\mathrm{BE}$ is now, as expected, lower than experiment. For the $\mathrm{C}(1 \mathrm{~s})$ core hole, static electron correlation effects are more important than they are for the $\mathrm{O}(1 \mathrm{~s}) \mathrm{BE}$ and only when they are accounted for the error in the BE has the expected negative sign. The final error for the CAS $(4,4) \mathrm{BE}$ allowing for relativistic corrections is $-0.42 \mathrm{eV}$. The difference with the XPS value is because dynamical correlation effects are not considered at the CASSCF level. Hence, as mentioned earlier on, the $\mathrm{CO}$ core level BEs provide an ideal test case for DFT methods.

Additional information regarding whether correlation effects in $\mathrm{C}(1 \mathrm{~s})$ and $\mathrm{O}(1 \mathrm{~s})$ BEs are treated in a balanced way can be gathered from the difference between the BE of the $\mathrm{O}(1 \mathrm{~s})$ and $\mathrm{C}(1 \mathrm{~s})$ core holes, $\triangle \mathrm{BE}$, defined in Eq. (2). It seems reasonable to argue that dynamical correlation effects for $\mathrm{C}(1 \mathrm{~s})$ and $\mathrm{O}(1 \mathrm{~s})$ would be rather similar. Indeed, $\triangle \mathrm{BE}$ computed by CAS $(4,4)$ calculations including relativistic effects is $246.44 \mathrm{eV}$, only $0.11 \mathrm{eV}$ larger than the experiment, $246.33 \mathrm{eV}$, and in much better agreement than the HF result, which differs by $1.25 \mathrm{eV}$ from the XPS data. Hence, for wave function based methods, the errors in the calculated $\triangle \mathrm{BE}$ give an estimate of the missing differential electron correlation effects.

Let us now focus on the $\mathrm{O}(1 \mathrm{~s})$ and $\mathrm{C}(1 \mathrm{~s}) \triangle \mathrm{SCF}$ BEs computed from DFT based methods. The situation regarding inclusion of static correlation effects in DFT methods may well be different from that discussed above for $\mathrm{HF}$ and CASSCF wave functions. This is because, beyond claiming that one is climbing Jacob's ladder, DFT methods lack a well-defined systematic way to improve the quality of the exchange-correlation potential. Table II compiles the results of the $\mathrm{O}(1 \mathrm{~s})$ and $\mathrm{C}(1 \mathrm{~s})$ core hole BEs as predicted for the fifteen DFT functionals analyzed in this work. Besides, the errors in the differences between the two computed BEs, $\triangle \mathrm{BE}$, are reported in the two rightmost columns of Table II. Errors with respect to experimental values, both without and with correction for relativistic effects, are reported.

Regarding the $\mathrm{O}(1 \mathrm{~s}) \mathrm{BE}$, the two leftmost columns of Table II show that, with the exception of the parameterized meta-GGA M11-L functional and its M11 range-separated hybrid counterpart, all errors are negative meaning that $\mathrm{BE}$ values are smaller than experiment. The GGA SOGGA11 functional gives a positive but very small error, $0.03 \mathrm{eV}$. However, the small error for SOGGA11-X is misleading since, at this point, the relativistic correction has not been added. When it is added, the error becomes $0.48 \mathrm{eV}$, still not very large but now clearly positive, suggesting that static correlation has not been quite properly treated. For the rest of the functionals considered here, the errors of the computed $\mathrm{O}(1 \mathrm{~s}) \mathrm{BE}$ with respect 
to experiment are of the same sign and order of magnitude than those corresponding to the HF or CAS $(4,4)$ method and of $\sim 1 \mathrm{eV}$. The one exception is the PBEsol functional which gives an $\mathrm{O}(1 \mathrm{~s}) \mathrm{BE}$ that is too small by over $3 \mathrm{eV}$. As we will discuss below, this functional also gives the poorest $\mathrm{C}(1 \mathrm{~s}) \mathrm{BE}$ which, in the view of the near-LDA character of PBEsol, is not so unexpected. However, it should be noted that the errors in the non-relativistic $\mathrm{O}(1 \mathrm{~s}) \mathrm{BEs}$ are comparable in magnitude to the relativistic correction for the $\mathrm{O}(1 \mathrm{~s}) \mathrm{BE}, 0.45 \mathrm{eV}$. Therefore, it is essential to include the relativistic corrections in order to make meaningful comparisons with experiment. Thus, in the following, we will stress comparisons between theory and experiment when the relativistic corrections are included in the theoretical results. Since the relativistic corrections shift the core level BEs to higher values, the errors also become larger in value. Thus in the usual case where the errors are less than zero, the effect of including the relativistic correction is often to reduce the magnitude of the error and to improve agreement with experiment; see Table II. However, notice that for some of the functionals studied, inclusion of relativistic effects changes the sign of the error, from being negative to becoming positive. Furthermore, the magnitude of the errors varies within the different types of functionals. It is noticeable that PBE, PBEsol, M11-L, and M11 perform especially worse than average and that meta-GGA functionals of the TPSS type clearly outperform the CAS $(4,4)$ result. Therefore, regarding the $\mathrm{O}(1 \mathrm{~s}) \mathrm{BE}$, for which static electron correlation effects are not as important as for the $\mathrm{C}(1 \mathrm{~s}) \mathrm{BE}$, once relativistic effects are accounted for, it can be concluded that DFT BEs exhibit an erratic behavior. This behavior makes it difficult to identify a pattern within a given family of functionals, nor is an improvement seen as the functional climbs Jacob's ladder. That means that even when dynamic correlation effects are the most important contribution to electronic correlation, it is not clear to which extent these contributions are covered by the different exchangecorrelation functionals. Finally, the exceptional good agreement between TPSS, TPSSm, and TPSSh calculated values for both $\mathrm{O}(1 \mathrm{~s})$ and $\mathrm{C}(1 \mathrm{~s})$ core hole BEs and experiment is remarkable, a feature that will be discussed further later on and which, nevertheless, points towards the right way to improve existing functionals beyond heavily fitting to experiment for a large database.

Next, we focus our attention on the computed errors for the $\mathrm{C}(1 \mathrm{~s})$ core hole BE, for which CASSCF calculations show that static electron correlation effects are more important. For most of the functionals studied, the computed BEs are larger than the measured XPS BE, giving rise to a positive sign of the BE error. Only PBE and M06 functionals and their hybrid counterparts underestimate the $\mathrm{BE}$. As in the case of the $\mathrm{O}(1 \mathrm{~s})$ core hole, the larger deviation with respect to experiment is found for PBE, PBEsol, M11-L, and M11 functionals. Again PBE and PBEsol largely underestimate the $\mathrm{C}(1 \mathrm{~s}) \mathrm{BE}$ values while M11L and M11 substantially overestimate them. The rest of the functionals display errors of similar size than the error obtained through CAS $(4,4)$ calculations. As for the $\mathrm{O}(1 \mathrm{~s})$ core level BE, TPSS, TPSSm, and TPSSh functionals also accurately describe the $\mathrm{C}(1 \mathrm{~s})$ core level $\mathrm{BE}$ which, in the view of the results discussed above, can be considered as an important result.
We already mentioned that absolute values of BEs are available for gas phase molecules through XPS measurements, but these are not straightforward to obtain for the XPS of condensed phase systems. In these cases, shifts of core level BEs between related systems are most often used. ${ }^{5-7}$ Hence, $\mathrm{BE}$ shifts, $\triangle \mathrm{BEs}$, are often computed to interpret experimental data. Here, we report the difference between the computed $\mathrm{BE}$ of the $\mathrm{O}(1 \mathrm{~s})$ and $\mathrm{C}(1 \mathrm{~s})$ core holes, $\triangle \mathrm{BE}$. Errors on the calculated $\triangle \mathrm{BE}$ with respect to experiment are presented in Table II. The BE shift, $\triangle \mathrm{BE}$, could be used as a criterion to establish if a given method is able to treat correlation effects in each core level in a balanced way. A small error in the computed $\triangle \mathrm{BE}$ can be interpreted as an indication that a functional is capable of describing the relevant effects of electronic correlation. Concerning the different DFT functionals analyzed here, it can be observed that only few of them lead to small errors in $\triangle \mathrm{BE}$. However, in some cases, a small error in the $\mathrm{BE}$ shift is accompanied by a very large error on the absolute values of $\mathrm{BE}$, see for instance, the M11 and PBE functionals, for which an acceptable error in $\triangle \mathrm{BE}$ is obtained, around $0.2 \mathrm{eV}$, despite that the $\mathrm{O}(1 \mathrm{~s})$ and $\mathrm{C}(1 \mathrm{~s}) \mathrm{BEs}$ are between 0.6 and $0.9 \mathrm{eV}$ off the expected values. Therefore, a small error in $\triangle \mathrm{BE}$ may ensure a balanced treatment of correlation effects but still fail to predict accurate absolute values because there are similar large errors for both the $\mathrm{O}(1 \mathrm{~s})$ and $\mathrm{C}(1 \mathrm{~s})$ BEs. A more overall balanced and accurate description is given by the TPSS, TPSSm, TPSSh, and even RevTPSS, with good relative $\triangle \mathrm{BE}$ and reasonable absolute BE values. Notice that M06$\mathrm{L}$ and hybrid GGA functionals result in satisfactory absolute errors for the $\mathrm{O}(1 \mathrm{~s})$ and $\mathrm{C}(1 \mathrm{~s}) \mathrm{BEs}$, but the errors in $\triangle \mathrm{BE}$ are much too large, meaning that these functionals do not properly account for the differential electron correlation effects between the $\mathrm{O}(1 \mathrm{~s})$ and $\mathrm{C}(1 \mathrm{~s})$ core holes. Finally, it is interesting to analyze the effect of the amount of exact Hartree-Fock exchange in the computed BEs for $\mathrm{O}(1 \mathrm{~s})$ and $\mathrm{C}(1 \mathrm{~s})$. For $\mathrm{C}(1 \mathrm{~s})$ $\mathrm{BE}$, the M06 functional, including $27 \%$ of exact exchange, gives a negative error of $-0.17 \mathrm{eV}$ including relativistic corrections, doubling the percentage of exact exchange increases the $\mathrm{BE}$ in such a way that now the error becomes positive by $0.45 \mathrm{eV}$. On the other hand, for $\mathrm{O}(1 \mathrm{~s}) \mathrm{BE}$, for which static correlation effects are less important, the amount of Hartree-Fock exchange does not affect the result. This fact can be a symptom that including the non-local Fock exchange term somehow mimics static electron correlation although in an unspecified manner.

From the results reported in Table II regarding the performance of the functionals studied here on the calculation of the $\mathrm{O}(1 \mathrm{~s})$ and $\mathrm{C}(1 \mathrm{~s})$ core hole BEs and $\triangle \mathrm{BEs}$, it can be concluded that there is no common trend within the functionals belonging to a given family, rather, the behavior turns out to be completely unpredictable. However, the functionals derived by Tao, Perdew, Staroverov, Scuseria, TPSS, TPSSm, and TPSSh, perform better than the rest, meaning that, at least for this particular case, these functionals are able to capture the relevant effects of static correlation. This is in line with a previous study on the performance of the TPSS, PBE, and HF methods on predicting core level binding energies of a set of 185 cores of 68 molecules containing main group elements where it was shown that the TPSS 
functional performs better than $\mathrm{HF}$ and $\mathrm{PBE}$ and with an overall mean absolute error of $\sim 0.2 \mathrm{eV}$ after including relativistic corrections or $\sim 0.3$ without them and, more importantly, similar values for the core level shifts with respect to a given reference. ${ }^{10}$

\section{CONCLUSIONS}

Several DFT methods comprising a variety of exchangecorrelation functionals of both local and non-local characters have been considered. The computed $\triangle \mathrm{SCF} C(1 \mathrm{~s})$ and $\mathrm{O}(1 \mathrm{~s})$ binding energies at the $\mathrm{C}-\mathrm{O}$ optimized distance and the corresponding binding energy shift for each method have been compared to the reported XPS experimental data.

For the $\mathrm{O}(1 \mathrm{~s})$ core hole BEs, inclusion of static electron correlation has a modest effect. On the other hand, correlation effects due to the $\mathrm{CO}(1 \pi)$ and $\mathrm{CO}(2 \pi)$ near degeneracy turn out to be more important for the final calculated $C(1 \mathrm{~s})$ core level $\mathrm{BE}$. The analysis of the BEs calculated with various exchangecorrelation functionals show an erratic behavior within the different groups of functionals and, therefore, tracing the origin of such behavior becomes extremely difficult. From the present results, a clear correlation with respect to the different types of functionals cannot be established. A small error of the difference between the $\mathrm{O}(1 \mathrm{~s})$ and $\mathrm{C}(1 \mathrm{~s}) \mathrm{BEs}, \triangle \mathrm{BE}$, with respect to experiment may indicate a balanced treatment of electron correlation effects but this is not enough to guarantee an accurate prediction of core level BEs since, in some cases, large undesirable errors in the absolute BEs are encountered. From the functionals studied here, the TPSS, TPSSm, and TPSSh functionals perform better than the rest and lead to both BEs and $\triangle \mathrm{BE}$ in comparable agreement with experiment than those obtained by CAS $(4,4)$ calculations which, in the view of the physical arguments on which these functionals are built, is encouraging.

Although, we have shown that the static correlation effects are treated quite accurately by these TPSS based functionals only for CO, there is every reason to believe that the TPSS based functionals will be successful for other cases where static correlation effects are important for XPS BEs. Indeed, this is in agreement with the results for a systematic study involving 185 cores of 68 molecules ${ }^{10}$ implying that the success of these functionals may also provide a basis for the analysis of the terms needed in the functional to properly describe XPS BEs.

\section{SUPPLEMENTARY MATERIAL}

See supplementary material for vibro-rotational spectroscopic constants for the ${ }^{12} \mathrm{C}^{16} \mathrm{O}$ molecule computed through Hartree-Fock (HF), CASSCF [CAS(4,4)], and various density functional theory (DFT) based methods are reported in Table S1 whereas Table S2 includes $\mathrm{O}(1 \mathrm{~s})$ and $\mathrm{C}(1 \mathrm{~s})$ core level binding energies and core level binding energy shifts for the $\mathrm{CO}$ molecule as obtained from $\triangle \mathrm{SCF}$ calculations using Hartree-Fock (HF), CASSCF [CAS(4,4)], and various density functional theory (DFT) based methods.

\section{ACKNOWLEDGMENTS}

P.S.B. acknowledges support from the U.S. Department of Energy, Office of Science, Office of Basic Energy Sciences, Chemical Sciences, Geosciences, and Biosciences (CSGB) Division through the Geosciences program at Pacific Northwest National Laboratory. C.S. and F.I. have been supported by the Spanish MINECO/FEDER CTQ-2015-64618-R grant and, in part, by Generalitat de Catalunya Grant Nos. 2014SGR97 and XRQTC. F.I. acknowledges additional support from the 2015 ICREA Academia Award for Excellence in University Research.

${ }^{1}$ K. Siegbahn, C. Nordling, A. Fahlman, R. Nordberg, K. Hamrin, J. Hedman, G. Johansson, T. Bergmark, S. E. Karlsson, I. Lindgren, and B. Lindberg, ESCA-Atomic, Molecular, and Solid State Structure Studied by Means of Electron Spectroscopy (Almqvist and Wiksells, Uppsala, Sweden, 1967).

${ }^{2}$ K. Siegbahn, C. Nordling, G. Johansson, J. Hedman, P.F. Hedén, K. Hamrin, U. Gelius, T. Bergmark, L. O. Werme, R. Manne, and Y. Baer, ESCA-Applied to Free Molecules (North-Holland, Amsterdam, 1969).

${ }^{3}$ C. S. Fadley, Surf. Interface Anal. 40, 1579 (2008).

${ }^{4}$ C. S. Fadley, in Electron Spectroscopy: Theory, Techniques and Applications, edited by C. R. Brundle and A. D. Baker (Academic Press, 1978), Vol. 2, p. 2.

${ }^{5}$ W. F. Egelhoff, Surf. Sci. Rep. 6, 253 (1987).

${ }^{6}$ P. S. Bagus, F. Illas, G. Pacchioni, and F. Parmigiani, J. Electron Spectrosc. Relat. Phenom. 100, 215 (1999).

${ }^{7}$ P. S. Bagus, E. S. Ilton, and C. J. Nelin, Surf. Sci. Rep. 68, 273 (2013).

${ }^{8}$ N. Pueyo Bellafont, P. S. Bagus, and F. Illas, J. Chem. Phys. 142, 214102 (2015).

${ }^{9}$ N. Pueyo Bellafont, F. Illas, and P. S. Bagus, Phys. Chem. Chem. Phys. 17, 4015 (2015).

${ }^{10}$ N. Pueyo Bellafont, F. Viñes, and F. Illas, J. Chem. Theory Comput. 12, 324 (2016).

${ }^{11}$ P. S. Bagus, C. Sousa, and F. Illas, J. Chem. Phys. 145, 144303 (2016).

${ }^{12}$ J. Cioslowski, Phys. Rev. A 43, 1223 (1991).

${ }^{13} \mathrm{P}$. Knowles, M. Schuetz, and H.-J. Werner, in Modern Methods and Algorithms of Quantum Chemistry, edited by J. Grotendorst (John von Neumann Institute for Computing, Jülich, 2000), Vol. 3, p. 97.

${ }^{14}$ E. Valderrama, E. V. Ludeña, and J. Hinze, J. Chem. Phys. 110, 2343 (1999).

${ }^{15}$ A. J. Cohen, P. Mori-Sánchez, and W. Yang, Science 321, 792 (2008).

${ }^{16}$ K. Sharkas, A. Savin, H. J. A. Jensen, and J. Toulouse, J. Chem. Phys. 137, 044104 (2012).

${ }^{17}$ W. L. Jolly, in Proceedings of the International Conference on Electron Spectroscopy, edited by D. A. Shirley (North-Holland, Amsterdam, The Netherlands, 1972), p. 629.

${ }^{18}$ W. L. Jolly and D. N. Hendrickson, J. Am. Chem. Soc. 92, 1863 (1970).

${ }^{19}$ D. P. Chong, J. Chem. Phys. 103, 1842 (1995).

${ }^{20}$ D. P. Chong, Chem. Phys. Lett. 232, 486 (1995).

${ }^{21}$ Y. Takahata and D. P. Chong, J. Electron Spectrosc. Relat. Phenom. 133, 69 (2003).

${ }^{22}$ R. S. Mulliken, J. Chim. Phys. 46, 497 (1949).

${ }^{23}$ B. O. Roos, P. R. Taylor, and P. E. M. Siegbahn, Chem. Phys. 48, 157 (1980).

${ }^{24}$ D. Cremer, Mol. Phys. 99, 1899 (2001).

${ }^{25}$ J. P. Perdew and K. Schmidt, AIP Conf. Proc. 577, 1-20 (2001).

${ }^{26}$ R. Peverati and D. G. Truhlar, Philos. Trans. R. Soc., A 372, 20120476 (2014).

${ }^{27}$ M. G. Medvedev, I. S. Bushmarinov, J. Sun, J. P. Perdew, and K. A. Lyssenko, Science 355, 49 (2017).

${ }^{28}$ H. Partridge, J. Chem. Phys. 90, 1043 (1989).

${ }^{29}$ J. P. Perdew, K. Burke, and M. Ernzerhof, Phys. Rev. Lett. 77, 3865 (1996).

${ }^{30}$ J. P. Perdew, A. Ruzsinszky, G. I. Csonka, O. A. Vydrov, G. E. Scuseria, L. A. Constantin, X. Zhou, and K. Burke, Phys. Rev. Lett. 100, 136406 (2008).

${ }^{31}$ R. Peverati, Y. Zhao, and D. G. Truhlar, J. Phys. Chem. Lett. 2, 1991 (2011).

${ }^{32}$ Y. Zhao and D. G. Truhlar, J. Chem. Phys. 125, 194101 (2006).

${ }^{33}$ R. Peverati and D. G. Truhlar, J. Phys. Chem. Lett. 3, 117 (2011).

${ }^{34}$ J. M. Tao, J. P. Perdew, V. N. Staroverov, and G. E. Scuseria, Phys. Rev. Lett. 91, 146401 (2003). 
${ }^{35}$ J. P. Perdew, A. Ruzsinszky, J. Tao, G. I. Csonka, and G. E. Scuseria, Phys. Rev. A 76, 042506 (2007).

${ }^{36}$ J. P. Perdew, A. Ruzsinszky, G. I. Csonka, L. A. Constantin, and J. Sun, Phys. Rev. Lett. 103, 026403 (2009).

${ }^{37}$ A. D. Becke, J. Chem. Phys. 98, 5648 (1993).

${ }^{38}$ C. Lee, W. Yang, and R. G. Parr, Phys. Rev. B 37, 785 (1988).

${ }^{39}$ C. Adamo and V. Barone, J. Chem. Phys. 110, 6158 (1999).

${ }^{40}$ R. Peverati and D. G. Truhlar, J. Chem. Phys. 135, 191102 (2011).

${ }^{41}$ V. N. Staroverov, G. E. Scuseria, J. Tao, and J. P. Perdew, J. Chem. Phys. 119, 12129 (2003).

${ }^{42}$ Y. Zhao and D. G. Truhlar, Theor. Chem. Acc. 120, 215 (2008).

${ }^{43}$ R. Peverati and D. G. Truhlar, J. Phys. Chem. Lett. 2, 2810 (2011).

${ }^{44}$ J. L. Dunham, Phys. Rev. 41, 721 (1932).
${ }^{45}$ C. Sousa, F. Illas, and P. S. Bagus, "Dependence of Core-Level Binding Energies on Bond Distance Reflect Bond Character" (unpublished).

${ }^{46}$ M. W. Schmidt, K. K. Baldridge, J. A. Boatz, S. T. Elbert, M. S. Gordon, H. S. J. Jensen, N. Koseki, K. Matsunaga, A. Nguyen, S. Su, T. L. Windus, M. J. Dupuis, and A. Montgomery, "General atomic and molecular electronic structure system," J. Comput. Chem. 14, 1347 (1993).

${ }^{47}$ F. Aquilante, L. De Vico, N. Ferré, G. Ghigo, P.-Å. Malmqvist, P. Neogrády, T. B. Pedersen, M. Pitonak, M. Reiher, B. O. Roos, L. Serrano-Andrés, M. Urban, V. Veryazov, and R. Lindh, "MOLCAS 7: The next generation," J. Comput. Chem. 31, 224-247 (2010).

${ }^{48}$ K. P. Huber and G. Herzberg, Molecular Spectra and Molecular Structure Vol. 4, Constants of Diatomic Molecules (Van Nostrand, New York, 1979).

${ }^{49}$ S. R. Smith and T. Darrah Thomas, J. Electron Spectrosc. Relat. Phenom. 8, 45 (1976). 